\title{
S18. Molecular neurobiology and mental disorders
}

MOLECULAR BIOLOGY -CHALLENGES OF PAST AND FUTURE Prof. Leena Peltonen

National Public Health Institute, Department of Human Molecular

Genetics, Mannerheimintie 166, FIN-00300 Helsinki, Finland.

The detailed information on human genome has been accumulation during past 20 years. Current technology allows us to analyze in detail this biological database which to large extent determines our development, maturation and aging including our predisposition to specific diseases. We know at present 2000 out of approximated 100000 human genes and the gene defect has been so far identified in close to 500 human diseases offering reliable diagnostics. Also paternity cases and other identification problems of individuals ean be solved reliably with gene analyses and expensive medicines can be produced at the low cost utilizing bacteria as factories which follow the orders dictated by human genes coding e.g. for a growth hormone or insulin.

Except diagnostics, the DNA technology allows identification of asymptomatic carriers of disease genes, with convenient DNA tests we can unequivocally identify the carriers of recessively inherited diseases and if such tests would be applied in population screenings, the birth of numerous severely affected children could be avoided every year.

The basic innovations, which have been crucial for the identification of gene defects in classical inherited diseases 1) the genome map milestoned by amplifiable markers 2) reliable identification of nucleotide changes and 3) advances in statistical analyses, will be cornerstones also in the dissection of the molecular background of diseases with complex genetic background, common at the population level. These diseases will be in the center of research efforts during the next decade. The possibility to identify predisposing genes also in psychiatric diseases is a foreseeable future and has risen a detate on the social and ethical consequences of such DNA analyses.
PSYCHIATRIC HYPOTHESES IN THE UGHT OF NEW MOLECULAR GENETIC DEVELOPMENT

M Ackenheil

Department of Neurochemistry, Psychiatric Hospital,

University of Munich, Nussbaumstr. 7, D-80336 Munich, Germany.

Family, twin and adoption studies show that genetic factors play a major role in the ethiology of the two major psychoses schizophrenia and manicdepressive illness. However, the mode of transmission is unclear and does not strictly follow Mendelian laws. The high discordance rate in twins and the low penetrance suggest environmental factors to play a role as well. Up to the 20th century the unitary or confinuum concepts of the major psychoses were dominating. The concepts correspond to the genetic term "anticipation" describing an inherited disorder with progressive earlier age of onset and increased severity in successive generations. - The intensive research of the genetics of psychiatric illnesses during the last decades led to a revival of these hypotheses. Arguments in favour are the occurrance of different psychiatric disorders in heavily affected pedigrees, the overlap of schizophrenic and affective syndromes, and the discordant findings in monocygotic twins. A higher incidence of schizophrenic disorders is found in the offsprings. Therefore, schizophrenic and affective disorders may be different forms of the same condition. - Recent development of molecular genetics delivers a biological explanation of anticipation and incomplete penetrance. The discovery of heritable unstable DNA sequences found in fragile $X$ syndrome, dystrophia myotonica (DM), and Huntington's Chorea (HC) has improved the knowledge of transmission of such heritable diseases. - The fragile $X$ syndrome, a genetic disorder with mental retardation and behavioural abnormalities, is associated with the rare fragile site at chromosome $X q 27.3$. Recently, the nature of the fragile $X$ mutation was uncovered at molecular levels showing an abnormal characteristic trinucleotide repeat (CCG) $n$. The tragile $X$ syndrome, like $D M$ is of unusual genetics. Normal families are stable and transmit the CCG repeat up to 40 copies like any other genetic polymorphism. However, a certain haplotype shows more coples than others. These haplotypes, are associated to e.g the fragile $X$ syndrome. Male and female fragile $X$ carriers have $70-200$ copies. After transmission from temaie to male an increase of the copies ( $>200$ ) can be observed which leads to the clinical expression of the syndrome. - The unstable sequence of the trinucleotide repeat could explain why different individuals exhibit different phenotypic components of a syndrome. The multifactorial inheritence of a syndrome can be explained as well. 


\section{INTERACTIONS OF RECEPTORS WITH PSYCHOTROPIC DRUGS \\ Olivier Civelli \\ F.Hoffmann-La Roche Ltd, CH-4002 Basel, Switzerland}

Neurotransmitters are able to interact at the synapse with three types of recognition molecules: the ligand-gated ion channels, the $G$ protein-coupled receptors and the uptake transporters. These molecules belong to respective supergene families, i.e. they share some sequence and/or structure similarities reflected mainly by a similar topological configuration. Most psychotropic drugs aim at modulating the interaction of a neurotransmitter with one of these recognition molecules.

Among the three supergene families of the neurotransmitter recognition molecules, the $G$ protein-coupled receptors are of particular importance in therapies because the dopamine D2 receptor, target of most anti-psychotic drugs, belong to this supergene family. Recently however, molecular cloning experiments have led to the discovery that two novel dopamine receptors, the D3 and D4 receptors, are closely related to the D2 receptors and also recognize neuroleptics. Furthermore it has been shown that the D3 and D4 receptor bind more efficiently some atypical neuroleptics than does the D2 receptor. In particular, the D4 receptor may be the preferential target of clozapine. Therefore the discoveries of these new receptors lead to a reevaluation of our understanding of antipsychotic drug-receptor interactions and may point at new targets for antipsychotic drug design programs.
COMPUTER MODELLING OF NEUROTRANSMITTER RECEPTORS AND NEW DRUG DESIGN

S.G. Dahl, Ø. Edvardsen, K. Kristiansen, I. Sylte

Department of Pharmacology, Institute of Medical Biology, University of Tromsø, N-9037 Tromsø, Norway.

The cloning of various neurotransmitter receptors has provided insight into their molecular structure and function. Knowledge about their 3-dimensional molecular structures might further explain receptor mechanisms, and would provide valuable tools for rational, structurebased drug design. No detailed 3-dimensional receptor structure has yet been reported from $\mathrm{x}$-ray crystallographic or NMR spectroscopic experiments. We have therefore used molecular modeling techniques to construct 3-dimensional models of 5- $\mathrm{HT}_{1 \mathrm{a}}, 5-\mathrm{HT}_{2 \mathrm{a}}, 5-\mathrm{HT}_{2 \mathrm{c}}$ and dopamine $D_{2}$ receptors. The models were based on the amino acid sequences of the receptors, and on a proposed analogy with the 3dimensional structures of bacteriorhodopsin and visual rhodopsin. The models were used to simulate agonist and antagonist receptor interactions, identify amino acid residues having particularly strong ligand interactions, and examine molecular mechanisms of agonistinduced receptor activation.

The molecular electrostatic potentials around the receptor models were mainly negative at the synaptic side and positive in the cytoplasmic domains. This indicates that negative electrostatic fields around certain synaptic domains may guide the positively charged neurotransmitter molecules, and receptor antagonists, towards their binding sites. The calculated molecular electrostatic potentials, and simulations of ligand-receptor interactions, demonstrated that it is essential to include the whole receptor protein, and not only the membrane spanning regions, in receptor models used for drug design. Rather than the traditional "lock and key" concept of ligand interactions, the simulations suggest dynamic "zipper" mechanisms, where flexible ligands bind to the receptor in several successive steps.

\section{ADENOVIRUS-MEDIATED GENE TRANSFER TO THE CENTRAL NERVOUS SYSTEM \\ J. Mallet, P. Hourellou *), G. Le Gal La Salle **), J.J. Robert, S. Berrard *) M. Perricaudet $\star \star \star)$ \\ *) L.G.N.C.N.R.S, 91190 Gif-sur-Yvette, France **) Institut A. Fessard C.N.R.S. Gif-sur-Yvette, France ${ }^{\star * *}$ ) Institut G. Poussy, C.N.R.S. Villejuif, France}

The efficient introduction of genetic material into quiescent nerve cells is important in the study of brain function and for gene therapy of neurological disorders. A replication-deficient adenoviral vector containing a reporter gene encoding $\beta$-galactosidase infected nerve cells in vitro and in vivo $\beta$-galactosidase was expressed in almost all sympathetic neurons and astrocytes in culture. Following stereotactic inoculations into the rat hippocampus and the substantia nigra, B-galactosidase activity was detected for over two months. Infected cells were identified as microglial cells, astrocytes, neurons using anatomical, morphological and immunohistochemical criteria. No obvious cythopathic effect was observed. We will discuss the implications of these findings to study the function of cloned genes and in the context of degenerative diseases, as an altemative to the grafting of fetal celis. 